\title{
Dielectric properties of aqueous solutions at normal and high dilutions
}

\author{
L.A. Morozova, S.V. Savel'yev \\ Kotelnikov Institute of Radio-Engineering and Electronics of the Russian Academy \\ of Sciences (Fryazino Branch), \\ Russia, 141190 Moscow oblast, Fryazino, Vvedenskogo pl. 1. \\ e-mail: mila-morozova.ludmila@yandex.ru
}

\begin{abstract}
Using an original method for measuring weak radiothermal electromagnetic signals, experimental data were obtained on the analysis of dispersions of noise attractors of the radiobrightness temperature of aqueous solutions of $\mathrm{NaCl}, \mathrm{Al}\left(\mathrm{NO}_{3}\right)_{3}$ at dilutions $\mathrm{C} 1(1: 100)-\mathrm{C} 12$ (12:100). The values of dispersions of noise attractors significantly differ from those for the control experiment with water. On the basis of the changed values of the noise attractors of the radiobrightness temperature, the values of the absorption coefficients of the solutions are calculated. The difference between the values of the solutions absorption coefficients and those for water is shown with a successive increase in their values with an increase in the degree of dilution. The difference between the values of the absorption coefficients of solutions of high dilutions and those for water is interpreted as a mechanism of cooperative interaction of ions with water molecules located outside the first hydration shell. A network of water molecules perturbed by ions contributes to the dielectric permittivity was obtained due to new properties at supramolecular interactions on the basis of dispersion interaction.
\end{abstract}

Keywords: radiothermal signals, radiobrightness temperature, normal and high dilution solutions, absorption coefficient, ion hydration shell, new dielectric properties, supramolecular interactions, dispersion interaction.

\section{Introduction}

The physical properties of aqueous solutions of electrolytes in the centimeter and millimeter ranges of electromagnetic waves (EMW), for example, [1, 2], attract some attention because there is a boundary between collective molecular processes in solutions, which are responsible for the statistical values of dielectric constants, and individual reactions of molecules of water due to the redistribution of $\mathrm{H}$-bonds between them and the mutual influence of molecules of water and substances dissolved in it. Waveguide methods for measuring the values of the dielectric 
permeability of aqueous solutions of sulfates depending on the concentration of dissolved substances with an emphasis on the decimeter range of EMW are considered in [3-6]. In these works, the hydration mechanism in solutions of lithium and sodium sulfates was taken into account and additional relaxation processes were identified, which were interpreted in the form of relaxation of ion pairs. In [6], on the basis of the results obtained, it was shown that the effective size of the hydration shell of the dissolved substance ions exceeds the limits of the first hydration shell. In [710], using the example of sulfates, it is noted that the stability of the hydration shells of ions is the result of either the formation of strong hydrogen bonds, which for an isolated ion manifests itself as the binding of water molecules with the formation of hydrogen bonds to stabilize the negative charge, or the formation of a hydration shell extending beyond the first hydration layer with a corresponding increase in relaxation time.

Based on the foregoing, the effect of dissolved ions on a network of water molecules can occur in different ways. In some cases, the cooperative interaction is limited to the formation of a single molecular hydration shell [8], which determines the dielectric properties of solutions. In other cases, ions that have an electric field with a higher intensity interact more significantly with a significantly larger number of water molecules than should be in the first hydration layer $[9,10]$. Thus, the analysis [10] suggests that ions with a high charge density can influence and cause the rotation of water molecules located far beyond the first hydration shell. Then, a supramolecular interaction of identically oriented water molecules can be created around the ions due to the mechanism of dispersion interaction.

These works, showing the importance of studying electrolyte solutions as model systems, were carried out in the centimeter EMW range. Therefore, it would be interesting to analyze the millimeter range of the frequency spectrum, where the absorption of EMW by solutions is determined by individual reactions of water molecules during the redistribution of H-bonds around the ions of a particular dissolved substance. The transition to the millimeter band of the EMW spectrum necessarily requires a change in the methodology for conducting experiments. To measure the properties of aqueous solutions, one can use a quasi-optical method for measuring absorbed and reflected plane electromagnetic waves, based on measuring the radiothermal radiation of solutions using ultra-high sensitive millimeter-wave radio receivers. This method was successfully applied in [11] and gave significant results for solutions with the standard concentration of the base substance, used in almost all works, with values above 1:20 (base substance/water).

Here it is necessary to notice that in the concentration range of the base substance from $30 \%$ to $1 \%$, which is usually mentioned in scientific publications, one can hardly expect the formation of stable multilayer hydration shells of ions, since 
even at $1 \%$ concentration, no more than 5 water molecules (on average) are located between the ions of the base substance in the solution. The formation of multilayer hydration shells around ions is possible only in solutions where, on average, tens of water atoms are located between the ions, which occurs in solutions of high dilution, for example, C3 (0.0001\%), and higher. In cases where there are on average 100 water atoms between the ions, the effect of the base substance ion on the multilayer hydration shell of another ion is insignificant. Such multilayer hydration shells of ions of dissolved substances, formed at high dilution, determine dielectric and pharmacological properties of solutions, which in practice differ from those expected in accordance with theory.

Proceeding from the results of recent studies showing that ions of substances can have hydration shells that are much larger than the first hydration layer, it is of interest to measure the physical characteristics of electrolyte solutions at concentrations less than $1 \%$. A decrease in the concentration of a dissolved substance will contribute to the formation of a hydration shell of each ion, which is not affected by other ions. In the case of successful registration of changes in the physical properties of solutions at high dilutions of base substances, it is possible to emphasize the significance of multilayer hydration shells of ions with sizes much larger than the hydration shells of ions for high concentrations. Interest in the formulation and solution of this problem is associated with the increasing use in traditional medicine of pharmacological preparations containing corresponding active substances in ultralow concentrations, for example, $[12,13]$, as well as works on modeling the action of such substances [14-17].

Thus, in [14], the presence of molecules of the initial substance (Immunoglobulin $\mathrm{G}$ ) was experimentally registered even after the procedure of its multiple sequential dilutions, which is confirmed by the light scattering method. Such residual molecules are associated with the density inhomogeneities of aqueous solutions. In [15], using the method of infrared emission spectroscopy, the results of intrinsic radiation of highly diluted solutions (IFN $\gamma$, antibodies to IFN $\gamma$, glycine buffer), as well as interferon-gamma solutions, are presented, showing the presence of significant particles. In [16], solutions of lactose saturated with BP AT (which is used to manufacture the drug) were studied. The analysis showed that at high dilutions, the solution is characterized by a wider distribution of the energies of intermolecular interaction of water molecules, and also contains more strongly bound water molecules compared to control samples. The studies were carried out by THzand IR-spectroscopy and indicated the presence of particles containing base substances in solutions of high dilutions. These results are supported by the analysis described in [17], where it is presented using THz-spectroscopy that structurally, BP $\mathrm{AT}$ are characterized by protein bands in $\mathrm{THz}$ spectra, and the mechanism of their 
effect on the target conformation is realized through changes in the protein hydration shell.

Thus, at high dilutions, the presence of the effect of new properties of substances can be associated with the formation of hydrate shells near the ions of dissolved substances on the basis of dispersion interaction. Moreover, the water molecules of the hydration shells are involved in the cooperative action determined by the dissolved substances. This means that it is possible to create a method allowing registering changes in the physical properties of aqueous solutions at high dilutions, which will make it possible to study the mechanism of these changes.

In this paper, on the basis of original experimental studies of radiothermal radiation, it is shown that it is possible to register the change in radiobrightness contrasts of aqueous solutions with a change in the concentration of the base substance for solutions with normal and high dilutions and, as a result, to determine the dielectric properties of the solutions.

\section{Materials and methods}

The following substances were used to prepare solutions:

1. Sodium chloride, $\mathrm{NaCl}$, extra-pure grade, STP TU KOMP 3-47811

2. Aluminum nitrate, $\mathrm{Al}\left(\mathrm{NO}_{3}\right)_{3}$, certificate of analysis: VWR $\mathrm{BDH}$ CHEMICALS, material 21050.260, grade analytical reagent, lot 19D024111.

To obtain the initial solutions and all samples of each substance with subsequent dilutions, water of the 1st purity class was used, with a resistance of 17.5 $\mathrm{M} \Omega / \mathrm{cm}$ (at the moment of such water production), produced using an Aquapur MLW ion exchanger (VEB MLW LABORTECHNIK 85005, ILMENAU, Germany).

Samples with a normal $135 \%$ concentration of base $\left(\mathrm{NaCl}\right.$ and $\left.\mathrm{Al}\left(\mathrm{NO}_{3}\right)_{3}\right)$ substances and their high and ultra-high dilutions were prepared by weighing with an accuracy of $0.1 \mathrm{~g}$. Solutions with a normal concentration of substances were prepared by weighing certain additions with their subsequent dissolution in distilled water.

High dilution samples were prepared immediately before measurements in the laboratory where these measurements were carried out. Solutions were made by serial dilutions 1:100. The initial dilution $\mathrm{C} 1(1: 100)$ was carried out in a $200 \mathrm{ml}$ vial, where $198 \mathrm{ml}$ of water was added to $2 \mathrm{ml}$ of the base substance, after which the vial was intensively shaken (potentiated) for 60 seconds. Further, the portion of the obtained solution (after the dilution C1) in an amount of $2 \mathrm{ml}$ was added to another vial with water (in an amount of $198 \mathrm{ml}$ ) and a similar potentiation was carried out, thus, the 
dilution C2 (1:100:100) was provided. Subsequent dilutions (C3-C12) were carried out similarly.

Before each measurement, the test solution was potentiated using a laboratory shaker THYS 2 MLW, Medizin-u, LABORTECHNIK \#72375, Leipzig, Germany (shaking frequency: $5 \mathrm{~Hz}$, amplitude: $100 \mathrm{~mm}$, shaking time: 60 seconds). Potentiation using the shaker was carried out along the vertical axis of vials. The potentiation procedure was performed in $250 \mathrm{ml}$ glass vials with Teflon screw caps. Each vial contained $200 \mathrm{ml}$ of the sample to be measured.

The measurement of the physical properties of solutions was carried out using a highly sensitive EMV radiometer at the fixed frequency $v=61.2 \mathrm{GHz}$ [18]. The characteristics of the radiometer were as follows: the accuracy of setting and maintaining the local oscillator frequency was $100 \mathrm{MHz}$; the input bandwidth 100 $\mathrm{MHz}$; the fluctuation sensitivity of the receiver is up to $0.15^{\circ} \mathrm{K}$ at a time constant of 1 sec, the minimum value of the power measured by the radiometer was $4.1 \cdot 10^{-16} \mathrm{~W}$ [18]. Before measuring solution samples, the radiometer was calibrated using a blackbody at three temperatures $\left(273,296\right.$ and $\left.373{ }^{\circ} \mathrm{K}\right)$, the temperature of ice melting, the temperature of the experiment and the boiling point of water.

\section{Results and discussion}

The interaction of aqueous solutions with EMW is determined by their dielectric properties. For example, the absorption coefficient for power at the frequency $v$ can be written using the Fresnel equations. With a normal incidence of EMW, this can be defined as:

$$
k(v)=1-\left|\frac{\sqrt{\varepsilon^{*}(v)}-1}{\sqrt{\varepsilon^{*}(v)}+1}\right|^{2},
$$

here $\varepsilon^{*}(v)$ is a complex dielectric permeability of the solution at the same frequency, which can be represented as two components [15]:

$$
\varepsilon^{*}(v)=\varepsilon^{\prime}(v)+i \varepsilon^{\prime \prime}(v),
$$


$\varepsilon^{\prime}(v)$ - dielectric permeability, $\varepsilon^{\prime \prime}(v)$ - dielectric losses, $i=\sqrt{-1}$. In the case of nonelectrolyte solutions, the dielectric losses are determined by the dipole relaxation of solution molecules. In the case of electrolyte solutions, dielectric losses are determined by two EMW absorption mechanisms. The first mechanism is associated with the reorientation of water molecules, which are dipoles, the second is responsible for the displacement of charged ions formed during the dissolution of a substance. Then, for electrolyte solutions, the EMW absorption is determined by the total losses:

$$
\varepsilon^{\prime \prime}(v)=\varepsilon_{d}^{\prime \prime}(v)+\varepsilon_{i}^{\prime \prime}(v),
$$

here $\varepsilon_{d}^{\prime \prime}(v)$ are the dipole and $\varepsilon_{i}^{\prime \prime}(v)$ are ion losses during EMW absorption. The paper [11] presents experimental results showing how dipole and ion losses manifest themselves in salt solutions at the frequency $v=61,2 \mathrm{GHz}$. It is obvious that solutions of potassium and cesium sulfates $\left(\mathrm{K}_{2} \mathrm{SO}_{4}\right.$ and $\left.\mathrm{Cs}_{2} \mathrm{SO}_{4}\right)$ at different concentrations have significantly different radiobrightness temperature, which makes it possible to clearly measure the values of their radiobrightness parameters, which are unambiguously related to the values of dielectric characteristics of the solutions, in the concentration range from 1 to $35 \%$.

The ability to significantly distinguish between the values of radiobrightness temperatures of the investigated solutions makes it possible to calculate the values of the absorption coefficients at the frequency of the experiment $v$ :

$$
k(v)=T_{R} / T,
$$

here $T_{R}$ is the experimentally measured radiobrightness temperature of the solution, $T$ is the thermodynamic temperature.

In this work, using the radiothermal method for measuring the radiation of aqueous solutions of substances, the radiobrightness temperatures of $\mathrm{NaCl}$ and $\mathrm{Al}\left(\mathrm{NO}_{3}\right)_{3}$ solutions at high (1-35\%) concentration of base substances are experimentally measured and the absorption coefficients are calculated for a comparative analysis with solutions of sulfate salts $\mathrm{K}_{2} \mathrm{SO}_{4}$ and $\mathrm{Cs}_{2} \mathrm{SO}_{4}$ due to using the results of [11]. 
Fig. 1 shows absorption coefficient $(k)$ curves for solutions of sulfates $\mathrm{Cs}_{2} \mathrm{SO}_{4}$ and $\mathrm{K}_{2} \mathrm{SO}_{4}$ (black curves 1 and 2), obtained using the data of [11], and solutions of $\mathrm{NaCl}$ and $\mathrm{Al}\left(\mathrm{NO}_{3}\right)_{3}$ (red curves 1 and 2), calculated from the measured radiobrightness temperatures.

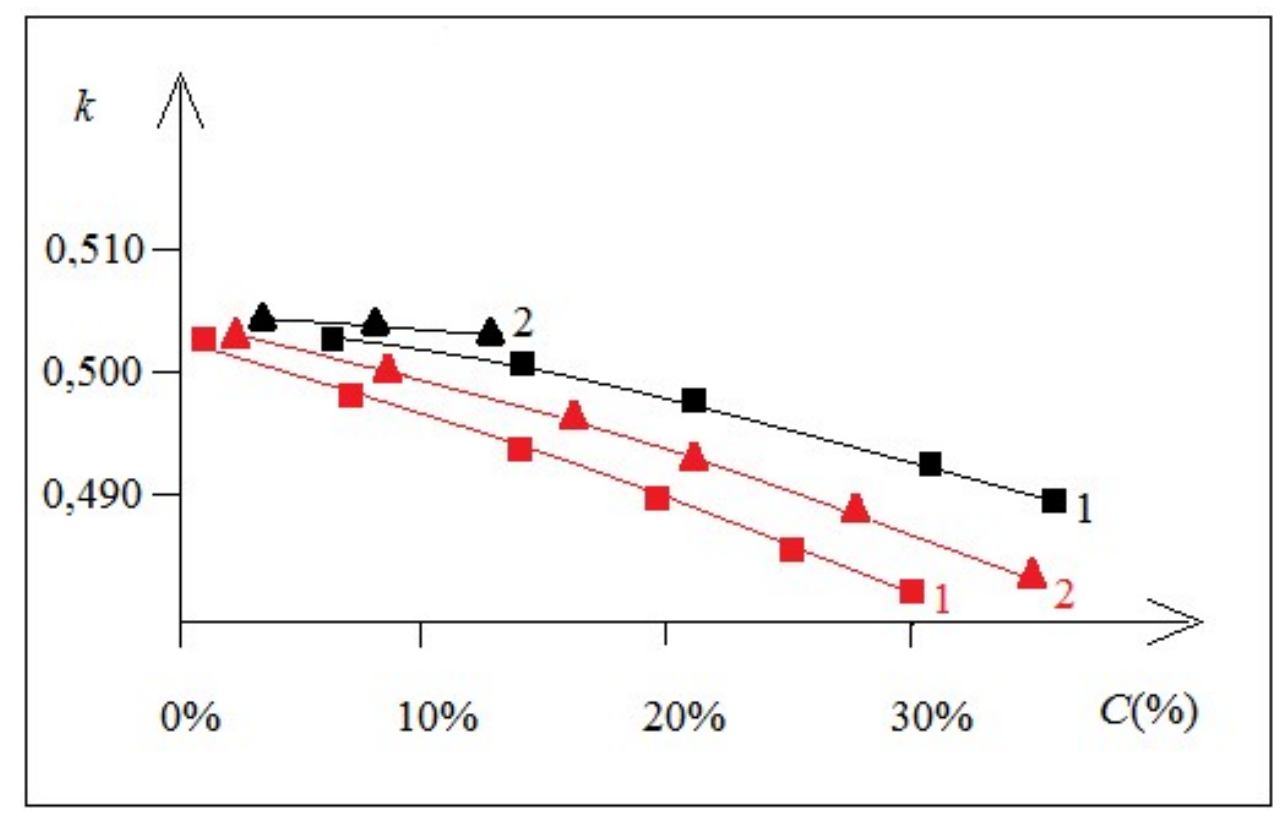

Fig 1. Dependence of absorption coefficient of $\mathrm{Cs}_{2} \mathrm{SO}_{4}$ and $\mathrm{K}_{2} \mathrm{SO}_{4}$ solutions (black curves, $\mathrm{K}_{2} \mathrm{SO}_{4}-1, \mathrm{Cs}_{2} \mathrm{SO}_{4}-2$ ) vs $\mathrm{Al}\left(\mathrm{NO}_{3}\right)_{3}$ and $\mathrm{NaCl}$ solutions (red curves, $\left.\mathrm{Al}\left(\mathrm{NO}_{3}\right)_{3}-1, \mathrm{NaCl}-2\right)$.

Fig. 1 shows that the dynamics of changes in the absorption coefficients of the solutions of the substances presented is similar, despite the different affinities of their ions for water. So, in the case of a solution of cesium sulfate $\mathrm{Cs}_{2} \mathrm{SO}_{4}$, the absorption coefficient of the solution, which determines the intrinsic radiation of the solution, falls, in the case of a solution of potassium sulfate $\mathrm{K}_{2} \mathrm{SO}_{4}$, the values of the absorption coefficient differ slightly from those for pure water, although it has a similar tendency to change the dielectric properties. In the case of $\mathrm{Al}\left(\mathrm{NO}_{3}\right)_{3}$ and $\mathrm{NaCl}$ solutions, the values of absorption coefficients decrease faster than those of sulfates when passing from water to solution. It follows from Fig.1 that changes in the absorption coefficients of solutions of the presented substances, in the general case associated with two mechanisms of interaction with EMW on the basis of dipole and ion losses, in the cases presented at high concentrations demonstrate the prevalence of the mechanism of dipole absorption of EMW. 
During the transition to solutions of substances with high dilutions, the difference between radiobrightness contrasts of the solutions decreases with a successive transition from one solution to another. In this case, the authors used the following original approach. For the millimeter range, the spectral density of thermal radiation of solutions is determined by the Rayleigh-Jeans Law:

$$
R_{0}=\frac{2 \pi}{\lambda^{2}} K T_{R}
$$

here $\lambda$ is the radiation wavelength, $K$ - Boltzmann constant, $T_{R}$ - radiobrightness temperature. The factor $G_{n}=K T_{R}$ is the thermal noise power spectral density and is directly proportional to temperature. An increase in the signal reception time constant $\tau$ in the experiment leads to the accumulation of a noise signal, then the radiobrightness temperature can be presented as follows:

$T_{R}=T_{R D}+\kappa T_{R A}(\tau)$,

here $T_{R D}$ is the constant component chosen so that $T_{R A}(\tau)$ is greater than 0 for the selected $\tau, T_{R A}(\tau)$ - the time-dependent variable component of the radiobrightness temperatures, $\kappa$ - the coefficient determined during the calibration of the receiving equipment before each measurement. For the same time constants, the variable component of the radiobrightness temperature is a function of the composition of a particular solution. To reveal the value of the variable component of the mentioned temperature in experiments with high dilution solutions, computer processing of the radiobrightness temperature noise track measured over a long time interval $n \tau$, $n \geq 1000$ was carried out.

The experimental procedure was as follows. The radiometer antenna was a horn with identical opening angles of $15^{\circ}$, a total length of $42 \mathrm{~mm}$, and a flange thickness of $2.5 \mathrm{~mm}$. The cuvette with the solution was a cube with a size of $70 \mathrm{~mm} \mathrm{x}$ $70 \mathrm{~mm} \times 70 \mathrm{~mm}$, which was completely filled. The radiometer horn reached the upper surface of the aqueous solution so that the line of contact with the solution passed along the entire perimeter of the horn edge; as a result, there was no need for an additional wall separating the antenna and the sample. Thus, at a radiometer reception wavelength of $4.9 \mathrm{~mm}$, the dimensions of the cuvette and the conditions of the experiment allow to assume that the absorption coefficient was measured taking into account the half-space dimensions of the samples.

The radiometer was an EMW receiver connected to a personal computer (PC), which recorded and processed the signal received from it. The radiometer generated a low-frequency signal, the level of which corresponded to the radiobrightness temperature of the measured sample. The signal was recorded in such a way that the time of the measurement was plotted along the abscissa axis, and the voltage from the 
radiometer output $U(v)$ was plotted along the ordinate axis. An example of the considered functional dependence is shown in Fig. 2 for a black body and C1-C3 dilutions solutions of $\mathrm{Al}\left(\mathrm{NO}_{3}\right)_{3}$. The measurement of the radiobrightness temperature of each sample was carried out for 30 minutes.

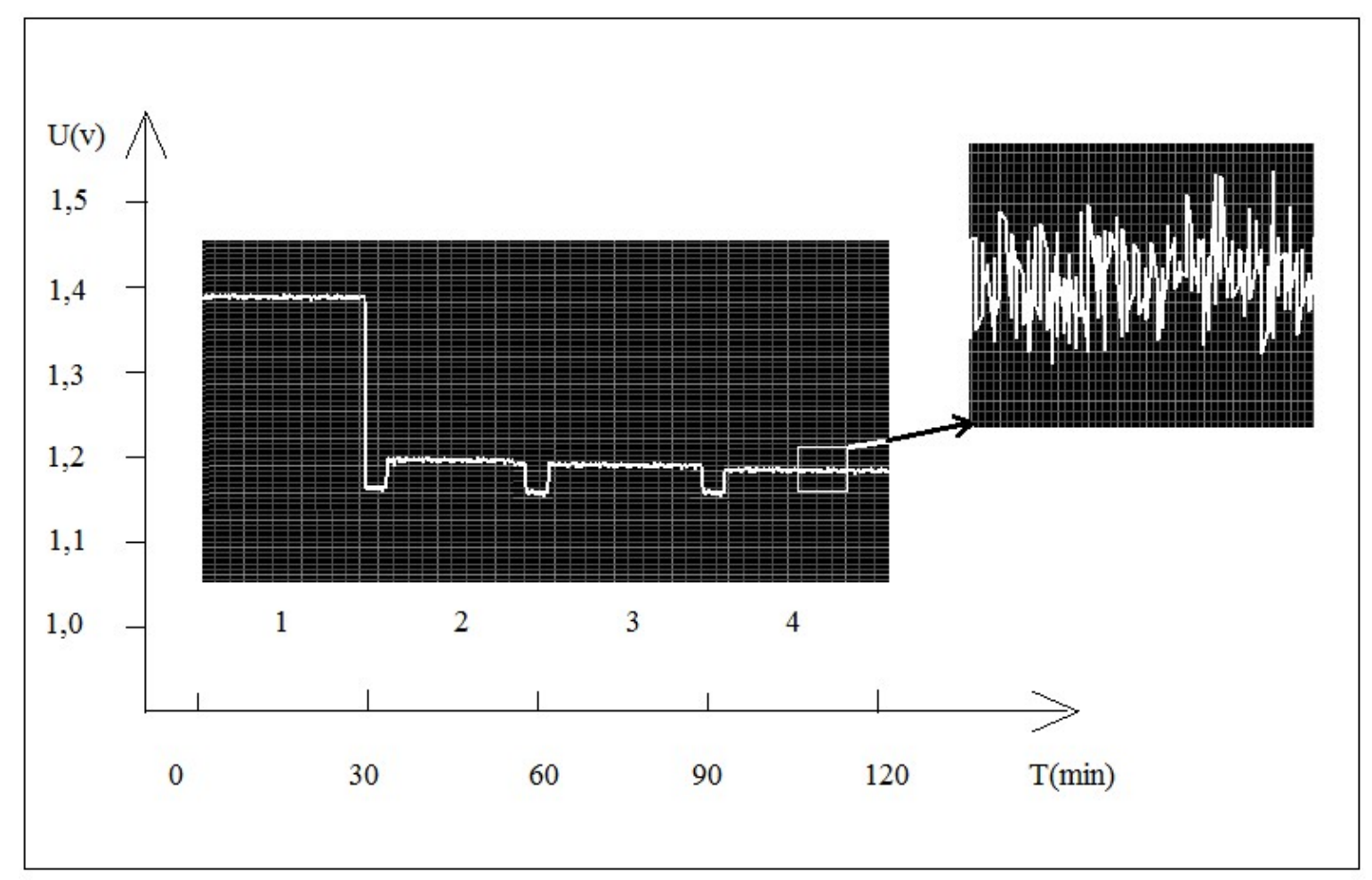

Fig. 2. Voltage at the radiometer output for a black body (time interval 1) and $\mathrm{C} 1-$ $\mathrm{C} 3$ dilutions solutions of $\mathrm{Al}\left(\mathrm{NO}_{3}\right)_{3}$ (time intervals 2-4)

Fig. 2 shows the differences in the values of radiobrightness contrasts of solutions of substances in the form of noise tracks lasting 30 minutes for each solution. At the end of the measurements, each noise track was processed to calculate the dispersion of the noise attractor for each measurement using Excel 2013 tabs. For the time constant $\tau=1 \mathrm{sec}$ of the low-frequency path of the radiometer, the number of measurement readouts for 30 minutes $n=1800$. For each solution sample, 6 measurements were performed. The calculated average values of the dispersions of noise attractors of the variable component of radiobrightness temperature for each measurement were entered into Excel 2013 tabs, with the help of which the average value of the dispersion of the noise attractors for all measurements of each sample and their standard deviation were calculated. 
To control the procedure for determining the significance of dispersions of noise attractors $T_{R A}(\tau)$ of solutions, control measurements of dispersions for samples prepared as described above, where water was used as the base substance, were carried out as part of the work. The dynamics of dispersions of noise attractors for the studied solutions of substances and water is presented in Fig. 3.

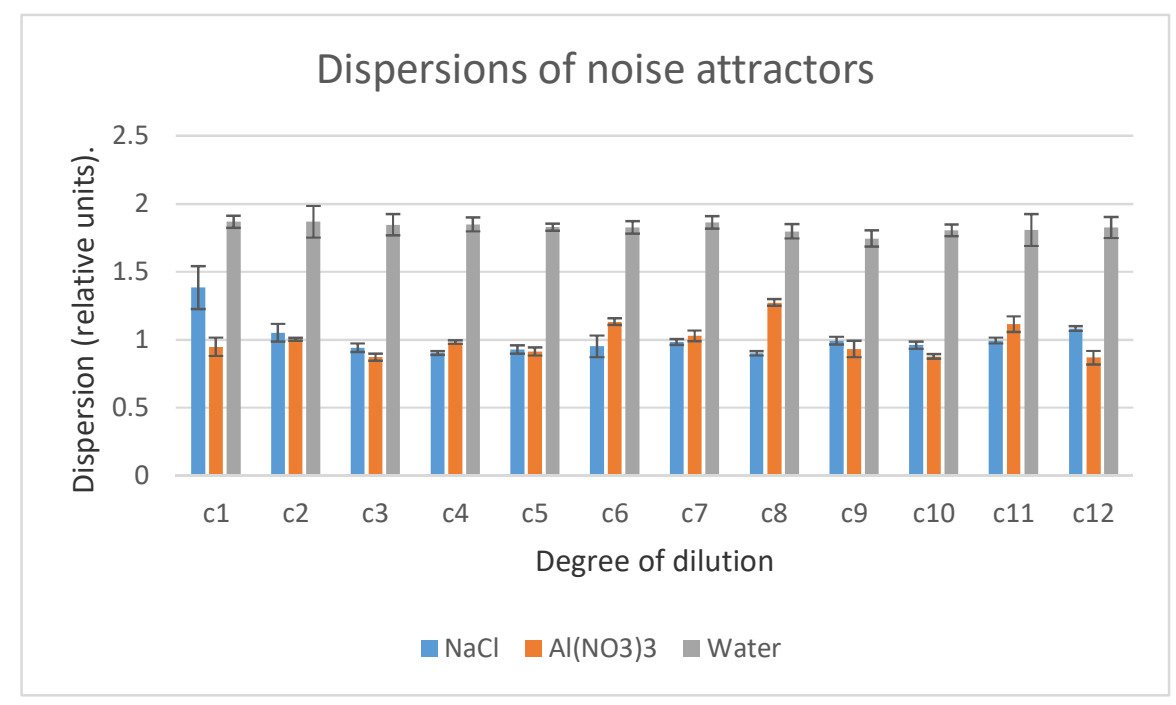

defined.

Error! Bookmark not

Fig. 3. Dispersions of noise attractors $T_{R A}(\tau)$ of solutions for high dilutions of $\mathrm{Al}\left(\mathrm{NO}_{3}\right)_{3}, \mathrm{NaCl}$ and water

The results obtained show the difference between the dispersion $T_{R A}(\tau)$ values of the studied solutions and water. The dispersions of noise attractors of solutions of the studied substances have values lower than in the control experiment. Thus, the method of radiothermal measurement of ultraweak electromagnetic signals at a frequency of $61.2 \mathrm{GHz}$ demonstrates the possibility of detecting differences in radiobrightness contrasts of high dilution solutions in the course of experiments.

As can be seen from Fig. 3, the values of dispersions of noise attractors $T_{R A}(\tau)$ of solutions change with increasing dilution degree, differing significantly from those for water. It is the difference between the dispersion of solutions and the data of the control experiment that can indicate the developed cooperative interaction of ions and water molecules, and ion-water cooperative interaction in aqueous solutions of the studied substances affects the number of water molecules, which is much grater than the volume of the first hydration layer. New properties of substances in high dilution solutions can be associated with ionic supramolecular interactions due to dispersion forces of equally oriented water molecules. 
The dynamics of changes in the dispersions of the noise attractors of the radiobrightness temperatures of the studied solutions, shown in Fig. 3, indicates a significant size of the emerging stable ion-water cooperative structures based on molecular interactions due to Coulomb forces and occurrence of strong hydrogen bonds. Proceeding from the differences of values of solution dispersions, it is possible to assume that such associative cooperative structures are quite stable under external mechanical influences and can retain their properties when passing from one solution to another.

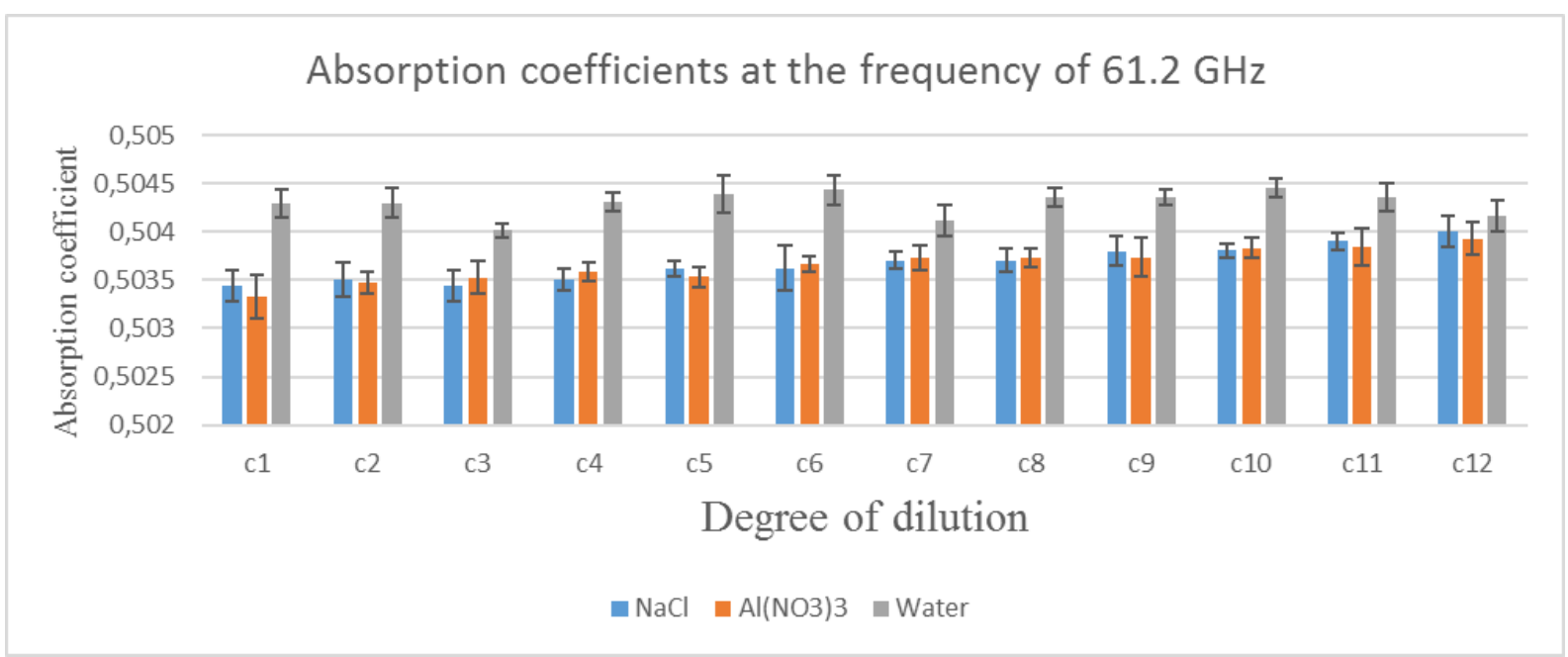

Fig. 4. Dynamics of absorption coefficient values for solutions of high dilution $\left(\mathrm{Al}\left(\mathrm{NO}_{3}\right)_{3}, \mathrm{NaCl}\right.$ and water $)$

Fig. 4 shows the dynamics of absorption coefficients for high dilutions of $\mathrm{Al}\left(\mathrm{NO}_{3}\right)_{3}, \mathrm{NaCl}$ and a control experiment with water, obtained taking into account (4) and (6) for the average values of dispersions of noise attractors $T_{R A}(\tau)$ of radiobrightness temperature. The dynamics of the absorption coefficient values indicates a monotonous increase in the values of the dielectric parameters with a change in the concentration of base substances, but they do not reach the values obtained for water, even at $\mathrm{C} 12$, which indicates a significant number of water molecules that are in cooperative interaction (determining the dielectric properties of solutions) with the ions of dissolved substances, and, therefore, participate in the creation of corresponding well developed hydration shells.

\section{CONCLUSIONS}

The presented experiments demonstrate the fundamental possibility of registering changes in the variable component of radiobrightness temperature for 
solutions of normal and high dilutions of base substances. It is shown that high dilution solutions of the studied substances are characterized by a difference in the dispersion of the noise attractor of the variable component of radiobrithness temperature. The individuality of the dielectric properties of solutions of substances at normal and high dilutions for some base substances is revealed. It is assumed that the presented dynamics of the dielectric properties of solutions at high dilutions is determined by the well developed hydration shell of ions, which significantly exceeds the dimensions of hydration shells of ions at normal concentrations of substances. New properties of substances in high dilution solutions may result from the construction of multilayer hydration shells based on supramolecular interactions around ions due to the manifestation of dispersion forces between water molecules involved in the cooperative interaction.

The work was carried out under a state contract. The authors would like to thank NPF Materia Medica Holding LLC.

\section{References}

1. Barthel J. Electrolyte Data Collection: Dielectric properties of water and aqueous electrolyte solutions. Chemistry data series V. 12. Part 2. DECHEMA, 1995.

2. Broadband Dielectric Spectroscopy / Eds. Kremer F., Schonhals A. Berlin: Springer-Verlag, 2003. $729 \mathrm{p}$.

3. Mungal A.G., Hart J. Measurement of the complex dielectric constant of liquids at centimeter and millimeter wavelengths. // Can. J. Phys. 1957. V. 35. P. 995-1003.

4. Buchner R., Capewell S.G., Hefter G., May P.M. Ion-Pair and Solvent Relaxation Processes in Aqueous $\mathrm{Na}_{2} \mathrm{SO}_{4}$ Solutions. // J. Phys. Chem. B. 1999. V.103. P. $1185-$ 1992.

5. Wachter W., Fernandez S., Buchner R. Ion Association and Hydration in Aqueous Solutions of $\mathrm{LiCl}$ and $\mathrm{Li}_{2} \mathrm{SO}_{2}$ by Dielectric Spectroscopy.// J. Phys. Chem. B 2007, 111, 9010-9017.

6. Shuang Liu, Guo-Zhu Jia, Shu Zhang. Consideration of fractal and ion-water cooperative interactions in aqueous $\mathrm{Na}_{2} \mathrm{SO}_{4}$ and $\mathrm{K}_{2} \mathrm{SO}_{2}$ solutions by dielectric relaxation spectroscopy// Physica A (2015), P. 1-8. http://dx.doi.org/10.1016/j.physa.2015.08.034.

7. X.-B. Wang, J.B. Nicholas, L.-S. Wanga. Electronic instability of isolated $\mathrm{SO}_{4}$ and its solvation stabilization. // J. Chem. Phys. 2000. V. 113. № 24. P. 10837-19840.

8. W. Wachter, W. Kunz, R. Buchner, G. Hefter, Is there an anionic Hofmeister effect on water dynamics? Dielectric spectroscopy of aqueous solutions of $\mathrm{NaBr}, \mathrm{NaI}, \mathrm{NaNO}_{3}, \mathrm{NaClO}_{4}$, and $\mathrm{NaSCN}$. // J. Phys. Chem. A 2005, 109, p. $8675-$ 8683. 
9. R.L. Wong, E.R. Williams. Dissociation of $\mathrm{SO}_{4}{ }^{2-}\left(\mathrm{H}_{2} \mathrm{O}\right) \mathrm{n}$ clusters, $\mathrm{n}=3-17$. // J. Phys. Chem. A 2003, 107, p. 10976-10983.

10. K. J. Tielrooij, N. Garcia-Araez, M. Bonn, H. J. Bakker, Cooperativity in ion hydration. // Science 328. 2010. P. 1006-1009. DOI: 10.1126/science.1183512 11. Lyashchenko A.K., Karataeva I.M., Dunyashev V.S. Relationship between radio brightness and dielectric properties of aqueous solutions of salts in the millimeter range of the spectrum. // Russ. J. Phys. Chem. A. 2019. V. 93. № 4. P. 552-557. 12. Epstein O.I., Pavlov I.F., Shtark M.B. Improvement of Memory by Means of Ultra-low Doses of Antibodies to S-100B Antigen. // Evidence-based Complementary and Alternative Medicine. 2006. Vol. 3, № 4. P. 541-545. 13. Epstein O.I. The Spatial Homeostasis Hypothesis. // Symmetry 2018, 10, 103. P. 1 - 14. https://dx.doi:10.3390/sym10040103

14. Nikolai F. Bunkin,* Alexey V. Shkirin, Barry W. Ninham, Sergey N. Chirikov, Leonid L. Chaikov, Nikita V. Penkov, Valeriy A. Kozlov, and Sergey V. Gudkov. Shaking-Induced Aggregation and Flotation in ImmunoglobulinDispersions: Differences between Water and Water-Ethanol Mixtures. // https://dx.doi.org/10.1021/acsomega.0c01444 15. Nikita Penkov, Nadezda Penkova. Analysis of Emission Infrared Spectra of Protein Solutions in Low Concentrations. // Frontiers in Physics. December 2020. V. 8. Art. 624779. P. 1 - 8. Doi: 10.3389/fphy.2020.624779/

16. Nikita Penkov, Eugeny Fesenko. Development of Terahertz Time-Domain Spectroscopy for Properties Analysis of Highly Diluted Antibodies. // Appl. Sci. 2020, 10, 7736; doi:10.3390/app10217736

17. Kristina N. Woods. New insights into the microscopic interactions associated with the physical mechanism of action of highly diluted biologics. // Scientific Reports. 2021. V. 11. Art. 13774. Doi.org/10.1038/s41598-021-93326-1 18. Krivoruchko V.I. Receiving radiometric module of $5 \mathrm{~mm}$ wavelength range with low noise amplifier at the input. // "Radiophysics and Quantum Electronics". 2003. V. TLVI. № 8-9. P. 782. 